\title{
VALIDAÇÃO ESTOCÁSTICA DE UM RECEPTOR RFID COM MÚLTIPLO ACESSO POR DIVISÃO DE CÓDIGO E PROCESSAMENTO POR DECOMPOSIÇÃO DE FATORES PARALELOS
}

\author{
F. H. J. B. TEIXEIRA, D. A. B. TAVARES E F. J. A. AQUINO \\ Instituto Federal de Educação, Ciência e Tecnologia do Ceará - IFCE \\ hitalojbraga@gmail.com*
}

Artigo submetido em março/2014 e aceito em agosto/2015

DOI: $10.15628 /$ holos.2015.2060

\section{RESUMO}

A aplicação de sistemas RFID (Identificação por Rádio Frequência) é uma importante ferramenta para o desenvolvimento e segurança dos processos industriais e afins. No entanto, o uso desta tecnologia em cenários com densas populações de transponders ainda não é trivial e muito menos comum em nosso cotidiano. $\mathrm{O}$ processo de singularização em ambientes com múltiplo acesso requer um sistema de controle eficiente e capaz de reduzir interferências, executando a distinção entre os vários sinais transmitidos no canal compartilhado. Neste trabalho, propomos ser válido o conceito de unicidade para um receptor RFID modelado com CDMA (Múltiplo Acesso por Divisão de Código) e o Algoritmo PARAFAC (Decomposição por Fatores Paralelos). Portanto a arquitetura consiste num tratamento de dados multilinear capaz de identificar e processar, simultaneamente, múltiplos transponders RFID a partir de um canal de rádio frequência compartilhado. $\mathrm{O}$ artigo apresenta uma introdução sobre os conceitos inerentes à pesquisa, bem como um conjunto de simulações para validar o modelo.

PALAVRAS-CHAVE: RFID, CDMA, Canais MIMO, Unicidade, Álgebra Multilinear e PARAFAC.

\section{STOCHASTIC VALIDATION OF A RECEIVER RFID WITH MULTIPLE ACCESS BY DIVISION CODE AND PROCESSING IN PARALLEL BREAKDOWN OF FACTORS}

\begin{abstract}
The application of RFID systems (Radio Frequency Identification) is an important tool for the development and safety of industrial and related processes. However , the use of this technology in scenarios with dense populations of transponders is not trivial and much less common in our everyday lives. The process of singling in environments with multiple access requires an efficient and capable of reducing interference control, running the distinction between the various signals transmitted on the shared channel. In this paper, we propose the
\end{abstract}

concept of being true uniqueness to an RFID receiver modeled with CDMA ( Code Multiple Access Division ) and PARAFAC algorithm (Decomposition for Parallel Factors ). Therefore, the architecture consists of a data processing multilinear able to identify and to process, simultaneously, transponders multiple RFID from a shared radio frequency channel. The article presents an introduction to the concepts inherent in research as well as a set of simulations to validate the model.

KEYWORDS: RFID, CDMA, MIMO channels, Uniqueness, e PARAFAC. 


\section{INTRODUÇÃO}

O RFID (Identificação por Rádio Frequência) é uma técnica de identificação baseada na comunicação através de sinais de rádio, onde é possível recuperar e armazenar dados remotamente a partir de um canal que liga os componentes do sistema. Segundo Mota (2006), a primeira grande utilização da tecnologia dessa foi durante a segunda guerra mundial quando as forças britânicas utilizaram a ferramenta para identificar amigos e inimigos, respondendo ou não aos pedidos de identificação por meio de ondas de rádio.

Os sistemas de identificação por frequência são compostos basicamente por um dispositivo leitor e pelos transponders. O leitor é responsável pela comunicação entre o sistema e os elementos externos ao processamento da informação e varia sua complexidade de acordo com as especificidades de cada aplicação. A Figura 1 ilustra os componentes básicos de um sistema RFID.

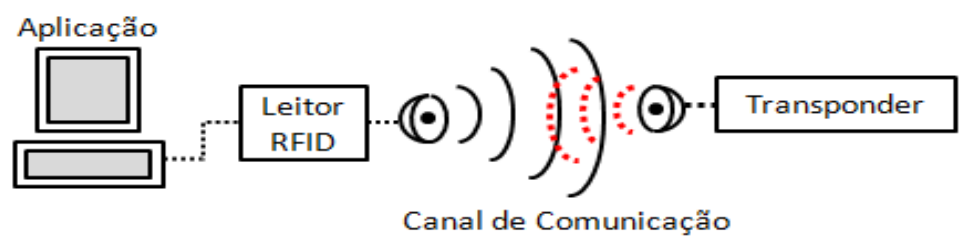

Figura 1: Componentes Básicos de um Sistema RFID.

Os transponders são dispositivos que viabilizam a comunicação entre os elementos a serem identificados e o leitor do sistema. Seu principal componente é um microchip que controla a comunicação com o leitor e possui uma memória interna onde são armazenados os dados. Os transponders podem ser classificados como ativos ou passivos. Os ativos possuem uma fonte de energia interna que alimenta seus circuitos integrados e fornece energia para a transmissão dos dados ao leitor. Já os passivos não possuem energia própria, devido a isto a alimentação dos seus circuitos embarcados é realizada a partir das ondas emitidas pela antena do dispositivo leitor.

Para Marques (2008), a aplicabilidade desses sistemas vem crescendo tangencialmente nos últimos anos, logo a necessidade de reconhecimento rápido de uma densa quantidade de transponders se tornou uma grande demanda para futuras pesquisas de desenvolvimento de arquiteturas RFID com esta otimização de processamento. Em aplicações como essa é fundamental que o processamento se dê em um curto espaço de tempo e com a menor probabilidade de erro possível. No entanto o problema para o desenvolvimento de uma arquitetura com estas características são as colisões múltiplas geradas pela transmissão dos sinais múltiplos. Devido a isso surge a necessidade do uso de métodos anti-colisão em sistemas RFID. Estes métodos gerenciam o acesso ao meio a partir de algoritmos de múltiplo acesso.

Um Receptor implementado com tecnologia CDMA (Acesso Múltiplo por Divisão de Códigos) e tratamento tensorial quando associado a um arranjo de antenas pode isolar os sinais do enlace dos diferentes elementos com maior facilidade, reduzindo os requisitos de controle de potência e tornando-se uma arquitetura interessante para o uso em RFID quando há necessidade de identificação de múltiplos transponders. Os sistemas com acesso múltiplo por divisão de códigos também são sensíveis às distribuições geográficas dos elementos nas células da antena inteligente associada ao sistema, podendo redirecionar sua área de cobertura para atender as áreas geográficas com maior densidade temporária de elementos/objetos. 
Este trabalho utiliza o algoritmo de Monte Carlo para a estimação estocástica da matriz de símbolos composta pelas informações transmitidas pelos transponders que compartilham o meio. Geramos entradas aleatórias a partir de um cenário RFID tipicamente real e simulamos os critérios de convergência e taxas de erros para a validação do modelo. O objetivo desta pesquisa é provar que a partir da unicidade inerente ao ordenamento tensorial é factível modelar uma arquitetura capaz de distinguir os dados transmitidos simultaneamente por diferentes transponders em canais RFID.

\subsection{Sistema com Múltilas Antenas (MIMO) em RFID}

O diferencial dos sistemas Multiple Input Multiple Output (MIMO) é o uso de múltiplas antenas, tanto para recepção, quanto para transmissão do dispositivo. A aplicação da tecnologia em portais RFID permite a criação de múltiplos canais no espaço viabilizando a partir de técnicas de decomposição por fatores o suo de múltiplas tags conforme a Figura 2.

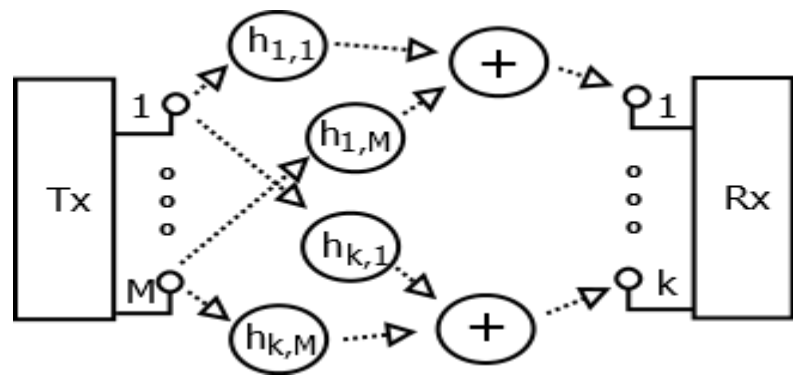

Figura 2. Canal com Múltilas Antenas (MIMO) em RFID.

em que $\left[H \in C^{K \times M}\right.$ ] é matriz de assinaturas espaciais, $\left[x \in C^{M \times 1}\right.$ ] é a matriz de símbolos transmitidos, $\left[\eta \in \mathrm{C}^{\mathrm{K} \times{ }^{1}}\right.$ ] é vetor de ruído gaussiano e $\left[\mathrm{y} \in \mathrm{C}^{\mathbf{M} \times{ }^{1}}\right.$ ] é o vetor de dados recebidos.

O objetivo da técnica é evitar que um sinal fortemente desvanecido de um transponder tenha uma única opção de recepção. Os canais em um sistema com múltiplas antenas sofrem desvanecimentos independentes por conta dos múltiplos percursos, portanto a técnica permite a multiplicidade de opções para um mesmo sinal. Portanto a grande vantagem dos sistemas MIMO está relacionada com o aumento da relação sinal-ruído (SNR) pela combinação de sinais de percursos independentes, viabilizando a diversidade de recepção para um mesmo sinal sem aumentar a utilização da banda disponível para o sistema.

\subsection{Desempenho dos sistemas com CDMA versus TDMA}

O CDMA (Múltiplo Acesso por Divisão de Código) é um método de acesso múltiplo no qual os usuários utilizam técnicas de espalhamento de espectral, ocupando toda faixa de frequências sempre que transmitem os dados. É executado na transmissão do sinal a partir de um código de espalhamento único para cada transponder, sendo este mesmo código utilizado no receptor para recuperar a sequência original dos dados. O sinal retro espalhado se assemelha a um ruído de faixa larga, o que reduz a interferência entre os sinais múltiplos viabilizando o acesso compartilhado.

Para Haykin (2008), a técnica possui diversas vantagens em detrimento aos demais métodos de múltiplo acesso. Dentre elas maior tolerância a interferências, maior tolerância a multipercursos e maior capacidade de alcance do sistema. 
A grande parte dos sistemas RFID utiliza dispositivos com tecnologia TDMA (Acesso Múltiplo por Divisão de Tempo) para obtenção do acesso ao meio. O princípio de funcionamento deste método é a divisão do tempo de envio total dos pacotes por tempos menores e proporcionais ao número de elementos do sistema. A Figura 3 faz uma comparação entre a transmissão RFID Multi-tag utilizando TDMA e CDMA.

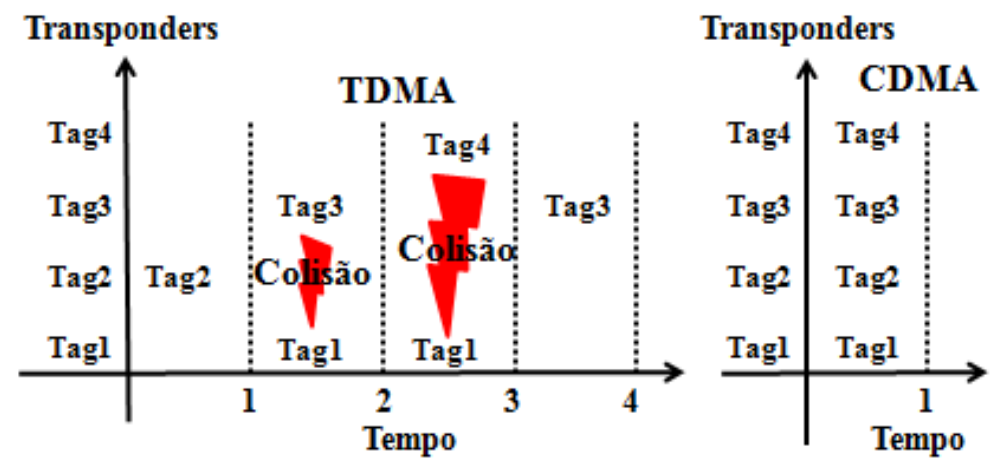

Figura 3: Transmissão RFID Multi-tag Utilizando TDMA e CDMA.

O uso de TDMA esta limitado ao tempo de time-slot do sistema, resultando em colisões quando há mais de uma tag transmitindo seus dados simultaneamente. Com o CDMA percebemos que esta limitação não afeta o sistema, já que os espectros de cada tag são alargados no espaço Tempo/Frequência e alocados em potência.

\subsection{Método de Monte Carlo (MMC)}

O método de Monte Carlo ( $\mathrm{MMC}$ ) pode ser descrito como um método estatístico no qual se utiliza uma sequência de números aleatórios para a realização da simulação de um cenário moldado com características reais. Portanto a aplicação do MMC consiste em descrever matematicamente um fenômeno físico a partir de um conjunto de equações que regem o funcionamento do sistema. Simulamos em software a estimação das matrizes de decomposição da arquitetura RFID com CDMA e decomposição por fatores paralelos (PARAFAC) gerando amostragens aleatórias de entrada com os mesmos parâmetros do processo real físico.

Conforme o número de iterações aumenta, melhora-se a convergência média do sistema, por conta da redução das incertezas estatísticas das grandezas envolvida na descrição matemática do modelo. Com isso esperamos obter uma estimativa do valor real à medida que um número suficientemente grande de amostragens é processado.

\section{MATERIAIS E MÉTODOS}

A criação de estratégias de processamento em domínios conjuntos agregou características híbridas ao tratamento de dados em sistemas de comunicação, integrando benefícios e limitações e expandido o alcance dos desempenhos de cada estratégia. O CDMA é uma destas arquiteturas híbridas e consiste no processamento Espaço-Código a partir da concatenação de conjuntos de diversidade e multiplicação numa estratégia única.

Segundo Lacerda Neto (2005), o tratamento da informação em domínios conjuntos duplos promoveu avanços importantes nos sistemas de comunicação, entretanto algumas limitações 
ainda impedem que haja uma recuperação fidedigna da informação. Dentre estas limitações podemos citar a não unicidade na estimação de canais e das sequências de dados transmitidos.

O tratamento multidimensional superior é a solução para o problema da não unicidade que sofre o tratamento de dados em domínios duplos. Portanto o objetivo da inserção de mais um domínio ao processamento da informação é agregar ao sistema as vantagens inerentes ao trato tensorial. Podemos definir uma estrutura tensorial como sendo um ordenamento multidimensional formado pela concatenação de matrizes.

\subsection{Ordenamentos Tensoriais de Dados e Reorganização Matricial}

A reorganização matricial dos tensores é necessária devido a razões matemáticas e computacionais. O processamento de dados matricial não implica em perdas de informação e, em nível de trato computacional e processamento da informação, está bem mais evoluído. Num tensor a informação é organizada pela alocação de cada elemento numa posição, para isto são utilizadas estruturas de ordem inferior, como vetores e matrizes, onde cada um destes elementos representara um subconjunto de informações do sistema.

Em Lacerda Neto (2005), há um estudo sobre uma das formas de reorganização matricial de um tensor denominada Forma Desdobrada (Unfolded). Esta técnica consiste na representação matricial do tensor a partir da concatenação dos seus diversos slices. É importante ressaltar que na reorganização por decomposição de fatores de menor ordem não há perda de dados, logo o conjunto de informações contido no tensor será o mesmo contido nas matrizes unfolded. A Figura 4.a ilustra uma representação dos slices horizontais, verticais e frontais de um tensor $\chi \in \mathrm{C}^{\mathrm{I} 1} \times \mathrm{I} 2 \times \mathrm{I} 3 \mathrm{de}$ ordem-3 respectivamente.

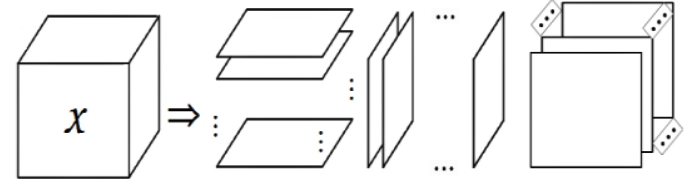

Figura 4.a. Slices de um tensor de ordem-3.
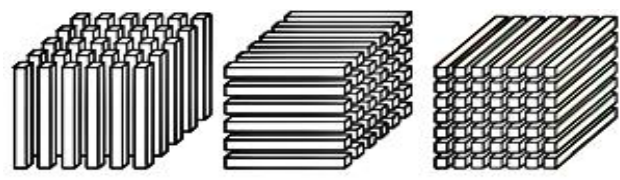

Figura 4.b. Fibras horizontais, verticais e frontais.

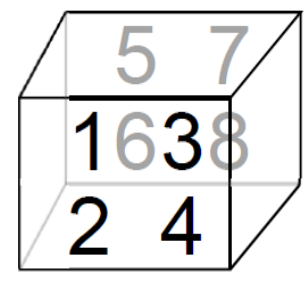

Figura 5. Tensor $\chi \in \mathrm{C}^{2 \times 2 \times 2}$

Os slices são compostos pelas fibras, que por sua vez são o análogo de ordem superior das linhas e colunas de uma matriz. A Figura 4.b ilustra as fibras que compõem os slices horizontais, verticais e frontais de um tensor de ordem-3 respectivamente. A ideia é fatiar as dimensões do tensor e organizar essas fatias uma ao lado da outra até sua totalidade. No caso de um tensor tridimensional, cada fatia é representada como uma matriz. A Figura 5 ilustra um exemplo de tensor $\chi \in C^{2 \times 2 \times 2}$ e as respectivas matrizes desdobradas $X_{(1)} X_{(2)}$ e $X_{(3)}$. 


$$
\begin{aligned}
& X_{(1)}=\left[\begin{array}{llll}
1 & 3 & 5 & 7 \\
2 & 4 & 6 & 8
\end{array}\right] \\
& X_{(2)}=\left[\begin{array}{llll}
1 & 2 & 5 & 6 \\
3 & 4 & 7 & 8
\end{array}\right] \\
& X_{(3)}=\left[\begin{array}{llll}
1 & 2 & 3 & 4 \\
5 & 6 & 7 & 8
\end{array}\right]
\end{aligned}
$$

Equação 1.a

Equação 1.b

Equação 1.c

\subsubsection{Produtos de Kronecker e Katri-Rao}

$\mathrm{O}$ produto de Kronecker entre duas matrizes $\mathrm{A} \in \mathrm{C}^{\mathrm{I} 1} \times \mathrm{I} 2$ e $\mathrm{B} \in \mathrm{C}^{\mathrm{I} 3 \times \mathrm{I} 4}$ fornece um vetor resultante do produto $A \otimes B$ de tamanho $\left[\left(I_{1} \times I_{3}\right) \times\left(I_{2} \times I_{4}\right)\right]$ e definido na Equação 2 .

$$
\mathrm{A} \otimes \mathrm{B}=\left[\begin{array}{cccc}
a_{11} B & a_{12} B & \ldots & a_{1 I_{2}} B \\
a_{21} B & a_{22} B & \ldots & a_{2 I_{2}} B \\
\vdots & \vdots & \ddots & \vdots \\
a_{I_{1} 1} B & a_{I_{1} 2} B & \ldots & a_{I_{1} I_{2}} B
\end{array}\right]
$$

Esta operação é uma generalização do produto externo entre duas matrizes de tamanho arbitrário. $\mathrm{O}$ produto de Kronecker resulta num vetor de ordem 3. Já o produto de Khatri-Rao entre duas matrizes $\mathrm{A} \in \mathrm{C}^{\mathrm{I} 1 \times \mathrm{J}} \mathrm{e} \mathrm{B} \in \mathrm{C}^{\mathrm{I} 2 \times \mathrm{J}}$, com o mesmo número de colunas, é uma matriz denotada por $A \diamond B$ e definida na Equação 2. O vetor resultante do produto de Katri-Rao é do tipo $C \in C^{I 1} \times \mathbf{I} 2 \times \mathbf{J}$, que por sua vez é um tensor formado pela concatenação das dimensões $I_{1}, I_{2}$ e J conforme a Equação 3 descreve.

$A \diamond B=\left(a_{1} \otimes b_{1} \ldots a_{k} \otimes b_{k}\right)$

Equação 3

\subsection{Unicidade}

O trato unicamente matricial não possui unicidade já que a decomposição de seus fatores sofre um efeito denominada ambiguidade de rotação. Este problema é gerado pelo efeito rotacional imposto as suas matrizes de decomposição que resulta em infinitas soluções para a decomposição do tensor. Portanto a unicidade não existe para o caso matricial, já que os vetores de decomposição podem sofrer rotação sem que isto afete sua estrutura. Consideremos um tensor de ordem dois, definido pela Equação 4.

Segundo Sidiropoulos (2004), a ambiguidade de rotação é gerada pelo fato de que qualquer matriz " $\mathrm{P}$ " não singular de dimensão $\mathrm{R} \times \mathrm{R}$ sofre ambiguidade de rotação de forma que a decomposição do tensor irá possuir infinitas soluções como representado pela Equação 5.

$X=\sum_{r=1}^{R} a_{i, r} b_{j, r}=A B^{T}$ Equação 4

$\mathrm{AB}^{\mathrm{T}}=\mathrm{APP}^{-1} \mathrm{~B}^{\mathrm{T}}$

Equação 5

A unicidade é um dos pilares de nossa arquitetura. Sem ela, seria impossível estimar precisamente as matrizes que compõe o tensor, pois sua decomposição não seria única. Partindo deste princípio sabemos que as matrizes de decomposição estimadas a partir do PARAFAC não 
são afetadas pelas ambiguidades de rotação, portanto não existe nenhum outro conjunto de matrizes que sejam capazes de reconstruir um mesmo tensor de dados.

\subsubsection{Condições de Kruskal}

Por definição sabemos que o posto de uma matriz é igual ao número de linhas linearmente independentes de uma matriz na forma escalonada. Segundo Kruskal (1977), um tensor $\chi$ composto por matrizes reais (,$B$ e $C$ ), terá a condição de unicidade satisfeita se a Equação 6 for respeitada.

$\mathrm{K}_{\mathrm{A}}+\mathrm{K}_{\mathrm{B}}+\mathrm{K}_{\mathrm{C}} \geq 2 \mathrm{R}+2$

Equação 6

em que $R$ é o número de fatores da decomposição PARAFAC e $k_{A}, k_{B}$ e $k_{C}$ são respectivamente os k-posto das matrizes $A, B$ e C. Logo quaisquer matrizes $\widetilde{A}, \widetilde{B}$ e $\widetilde{C}$ estão relacionadas com A, B e $C$ pela Equação 7.

$\widetilde{\mathrm{A}}=\mathrm{A} \prod \Delta_{1}, \widetilde{\mathrm{B}}=\mathrm{B} \prod \Delta_{2}$ e $\widetilde{\mathrm{C}}=\mathrm{C} \prod \Delta_{3}$

Equação 7

onde $\Pi$ é a matriz de permutação e $\Delta_{1}, \Delta_{2}$ e $\Delta_{3}$ são as matrizes diagonais que satisfazem a condição:

$\Delta_{1}, \Delta_{2}$ e $\Delta_{3}=\mathrm{I}_{\mathrm{M}}$

Equação 8

\subsection{Decomposição por Fatores Paralelos (PARAFAC)}

O ordenamento tensorial é baseado em conceitos de álgebra multilinear e consiste na decomposição única de cada dimensão do tensor, que por sua vez representa uma matriz de dados. Hoje é largamente utilizado em telecomunicações para a modelagem de sistemas de comunicação. Segundo Kolda \& Bader (2009), o modelo PARAFAC de um tensor $x \in C^{\text {I1 }} \times$ I2 $\times$ I3 é dado na forma de produto externo por:

$\chi=\sum_{\mathrm{r}=1}^{\mathrm{R}} \mathrm{a}_{\mathrm{r}} \circ \mathrm{b}_{\mathrm{r}} \circ \mathrm{c}_{\mathrm{r}}$ para $\mathrm{r}=1, \ldots \mathrm{R}$.

Equação 9

onde $a_{r} \in C^{\mathbf{I} 1}, b_{r} \in C^{I} 2$ e $c_{r} \in C^{I 3}$. Sendo $R$ o rank do tensor. Podemos ainda utilizar o produto de Kathi-Rao para representar a forma matriciada do tensor conforme as equações abaixo. Onde $D_{i_{1}}(\mathrm{~A})$, por exemplo, forma uma matriz diagonal com a $\mathrm{i}_{1}$-ésima linha de $\mathrm{A} \in \mathrm{C}^{\mathbf{I} 1 \times \mathbf{I} 2}$ na diagonal principal.

$\mathrm{X}_{(1)}=\left(\begin{array}{c}\mathrm{X}_{. .1} \\ \vdots \\ \mathrm{X}_{. . \mathrm{I}_{3}}\end{array}\right)=\left(\begin{array}{c}\mathrm{AD}_{1}(\mathrm{C}) \\ \vdots \\ \mathrm{AD}_{\mathrm{I}_{3}}(\mathrm{C})\end{array}\right) \mathrm{B}^{\mathrm{T}}=(\mathrm{C} \diamond \mathrm{A}) \mathrm{B}^{\mathrm{T}}$
$\mathrm{X}_{(2)}=\left(\begin{array}{c}\mathrm{X}_{1 . .} \\ \vdots \\ \mathrm{X}_{\mathrm{I}_{1} . .}\end{array}\right)=\left(\begin{array}{c}\mathrm{BD}_{1}(\mathrm{~A}) \\ \vdots \\ \mathrm{BD}_{\mathrm{I}_{1}}(\mathrm{~A})\end{array}\right) \mathrm{C}^{\mathrm{T}}=(\mathrm{A} \diamond \mathrm{B}) \mathrm{C}^{\mathrm{T}}$

Equação 10

Equação 11 
$\mathrm{X}_{(3)}=\left(\begin{array}{c}\mathrm{X}_{.1 .} \\ \vdots \\ \mathrm{X}_{. \mathrm{I}_{2}}\end{array}\right)=\left(\begin{array}{c}\mathrm{CD}_{1}(\mathrm{~B}) \\ \vdots \\ \mathrm{CD}_{\mathrm{I}_{2}}(\mathrm{~B})\end{array}\right) \mathrm{A}^{\mathrm{T}}=(\mathrm{B} \diamond \mathrm{C}) \mathrm{A}^{\mathrm{T}}$

onde $\mathrm{X}_{(1)}, \mathrm{X}_{(2)}, \mathrm{X}_{(3)}$ são respectivamente as matrizes desdobradas (unfolded) modo-1, modo-2 e modo-3 do tensor. A Figura 6 ilustra a formação de um tensor de rank-1 e ordem-3.

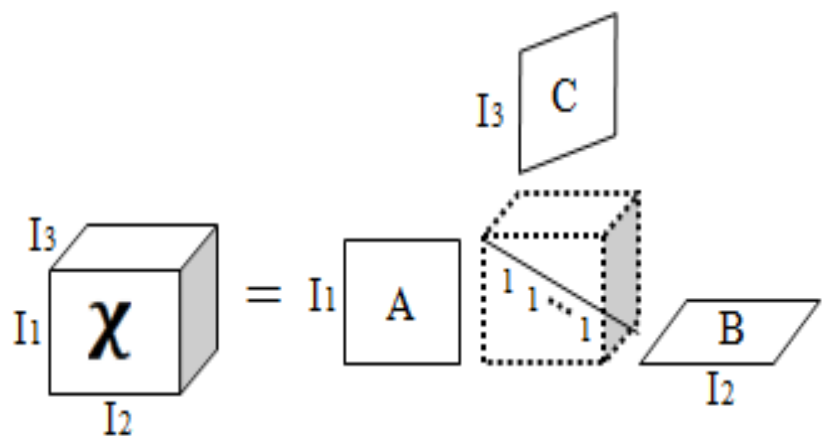

Figura 6. Decomposição PARAFAC de Ordem-3.

\subsection{Algoritmo de Mínimos Quadrados Alternados (ALS)}

O algoritmo ALS visa minimizar a soma do quadrado da diferença dos elementos do tensor de dados original e um tensor inicial estimado, que é gerado de maneira aleatória através do método estocástico de Monte Carlo. A técnica realiza a estimação iterativa e alternada das diversas matrizes de informação estimada até alcançar a mínima convergência esperada pelo modelo, fazendo com que o tensor estimado se aproxime cada vez mais do tensor original e que a diferença entre os dois tensores diminua a cada iteração do algoritmo.

O algoritmo consiste em minimizar a função de custo descrita na Equação 15 a partir das matrizes de decomposição do PARAFAC. Para facilitar o entendimento das simulações utilizaremos a notação descrita na Tabela 1 para as matrizes que compõem o modelo.

Tabela 1. Matrizes A, C e S.

\begin{tabular}{l}
\hline $\mathbf{A} \rightarrow$ Matriz de Assinaturas Espaciais (A) \\
\hline $\mathbf{C} \rightarrow$ Matriz de Códigos CDMA (B) \\
\hline $\mathbf{S} \rightarrow$ Matriz de Símbolos (C) \\
\hline
\end{tabular}

$f_{\text {custo }}={ }_{\mathrm{A}, \mathrm{C}, \mathrm{S}}^{\min } \sum_{\mathrm{n}=1}^{\mathrm{N}}\left\|\mathrm{Y}(\mathrm{n})-\mathrm{AD}_{\mathrm{n}}(\mathrm{S}) \mathrm{C}^{\mathrm{T}}\right\|_{\mathrm{F}}^{2}$

Equação 13

A convergência do algoritmo é atingida quando a diferença entre o tensor original e o tensor estimado não varia consideravelmente. Calcula-se o erro e (i)-ésima iteração a partir da Equação 16. Neste trabalho consideramos que a convergência do algoritmo será garantida quando $\left\|\mathrm{e}_{\mathrm{i}}-\mathrm{e}_{(\mathrm{i}-1)}\right\|=10^{-6}$ na i-ésima iteração.

$\mathrm{e}_{\mathrm{i}}=\sum_{\mathrm{n}=1}^{\mathrm{N}}\left\|\mathrm{Y}(\mathrm{n})-\mathrm{AD}_{\mathrm{n}}(\mathrm{S}) \mathrm{C}^{\mathrm{T}}\right\|_{\mathrm{F}}^{2}$

Equação 14 


\subsection{Desenvolvimento do Modelo RFID com CDMA e PARAFAC}

Para simulação de nosso modelo iremos considerar um cenário RFID CDMA uplink onde quatro (4) transponders comunicam-se simultaneamente com um portal leitor RFID, que por sua vez será composto por um arranjo de $\mathrm{K}$ Antenas Receptoras (AntRx). Iremos utilizar o algoritmo ALS-PARAFAC para realizar a estimação dos dados emitidos pelos transponders do sistema variando as características do cenário.

Consideremos ainda que a matriz de assinaturas espaciais do sistema é não dinâmica e que devido à técnica de Múltiplo Acesso por Divisão de Código (CDMA) a matriz de sequências de espalhamento espectral é única para cada transponder, podendo ser ainda conhecida na recepção do sinal conforme a técnica. O desempenho das estimações das matrizes será analisado a partir do Normalized Mean Square Error (NMSE) e pelo Bit Error Rate (BER) para diversos valores de Relação Sinal Ruído (SNR) conforme a Equação 15.

$\mathrm{NMSE}=\frac{1}{\mathrm{~W}} \sum_{\mathrm{W}=1}^{\mathrm{W}} \frac{\left\|\hat{\mathrm{A}}_{(\mathrm{w})}-\mathrm{A}\right\|_{\mathrm{F}}^{2}}{\|\mathrm{~A}\|_{\mathrm{F}}^{2}}$

Equação 15

onde $(\hat{A})$ é a matriz estimada de assinaturas espaciais na w-ésima realização de Monte Carlo.

A Figura 7 descreve os componentes básicos de uma estrutura RFID CDMA para cenários com múltiplas tags. Percebe-se que o Leitor emite uma onda que alimenta os circuitos embarcados de cada transponder, que por sua vez responde a este estimulo com seus dados retroespalhados. O processo de singularização da banda espalhada acontece no Rx do Leitor após o processo de estimação das informações. Cada tag possui um código único de espalhamento espectral que viabiliza o mutilo acesso ao Rx do dispositivo leitor.

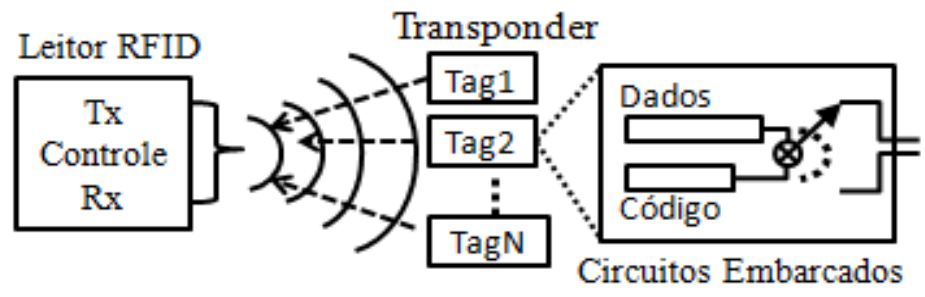

Figura 7: Sistema RFID com CDMA para Ambientes Multi-tag.

\subsection{Geração da Matriz de Códigos de Espalhamento Espectral}

Em nossa pesquisa as sequências de códigos que geram o espalhamento espectral para o método CDMA baseiam-se nas matrizes de Hadamard-Walsh. Estas são matrizes quadradas de tamanho $2^{n} \times 2^{n}$ com elementos \pm 1 , sendo as linhas e colunas construídas recursivamente e ortogonais entre si. Tomando $\mathrm{H}(4)$ temos quatro sequências de espalhamento espectral representadas por cada linha da matriz abaixo ilustrada na Equação 16.

$H_{(4)}=\left[\begin{array}{cc}H(2) & H(2) \\ H(2) & -H(2)\end{array}\right]=\left[\begin{array}{rrrr}1 & 1 & 1 & 1 \\ 1 & -1 & 1 & -1 \\ 1 & 1 & -1 & -1 \\ 1 & -1 & -1 & 1\end{array}\right]$

Equação 16 
Cada linha da matriz define uma sequência de Walsh e representa o complemento do código de acesso para as diferentes tags, que por sua vez são representadas pelas colunas da matriz. Como as sequências $S_{i}$ de $\left(H_{n}\right)$ são ortogonais entre si não há interferência entre sinais sincronizados transmitidos do mesmo transponder.

Suponhamos que um determinado transponder utilizando a sequência $S_{1}$ de $\left(H_{4}\right)$, [1111], queira transmitir a informação [1-1-1 1]. Vejamos no Quadro 1 como se dá o processo de espalhamento espectral tanto na transmissão quanto na recepção do sinal.

Quadro 1. Espalhamento Espectral $\rightarrow$ RFID Hadamard Walsh

\begin{tabular}{|c|c|c|c|c|}
\hline \multirow[t]{2}{*}{ Informação : } & 1 & -1 & -1 & 1 \\
\hline & $\otimes$ & $\otimes$ & $\otimes$ & $\otimes$ \\
\hline Seguência $\mathrm{S}_{1}$ de $\left(\mathrm{H}_{4}\right)$ : & 1111 & 1111 & 1111 & 1111 \\
\hline Informação Transmitida: & {$\left[\begin{array}{llll}1 & 1 & 1 & 1\end{array}\right]$} & {$[-1-1-1-1]$} & {$[-1-1-1-1]$} & {$\left[\begin{array}{llll}1 & 1 & 1 & 1\end{array}\right]$} \\
\hline \multirow[t]{2}{*}{ Informação Recebida: } & 1111 & $-1-1-1-1$ & $-1-1-1-1$ & 1111 \\
\hline & $\otimes$ & $\otimes$ & $\otimes$ & $\otimes$ \\
\hline Seguência $\mathrm{S}_{1}$ de $\left(\mathrm{H}_{4}\right)$ : & 1111 & 1111 & 1111 & 1111 \\
\hline Informação : & 1 & -1 & -1 & 1 \\
\hline
\end{tabular}

onde $\otimes$ representa o produto entre matrizes e $S_{1}$ de $\left(H_{4}\right)$ o código de espalhamento espectral gerado pela primeira sequência da matriz de Hadamard para 4(quatro) transponders.

A informação transmitida é o resultado do produto entre a informação original e a sequência $S_{1}$, gerando um espalhamento no espectro e fazendo com que o sinal seja transmitido utilizando uma banda muito maior, portanto otimizando a Relação Sinal Ruído (SNR). Além disso, o espalhamento espectral é gerado a partir de sequências ditas ortogonais entre si, logo o produto interno entre estas é sempre zero se as sequências forem diferentes, mas caso as sequências sejam iguais, ou seja, caso o receptor conheça o código de espalhamento então o produto gera a informação original. As Equações 17 e 18 representam o dito acima.

$\mathrm{R}_{\mathrm{xy}}=\sum_{\mathrm{i}=1}^{\mathrm{L}} \mathrm{x}_{\mathrm{i}} \cdot \mathrm{y}_{\mathrm{i}}=0$

Equação17

$\mathrm{R}_{\mathrm{xx}}=\sum_{\mathrm{i}=1}^{\mathrm{L}} \mathrm{x}_{\mathrm{i}} \cdot \mathrm{x}_{\mathrm{i}}=\mathrm{R}_{\mathrm{yy}}=\sum_{\mathrm{i}=1}^{\mathrm{L}} \mathrm{y}_{\mathrm{i}} \cdot \mathrm{y}_{\mathrm{i}}=\mathrm{L}$

Equação18

onde $x_{i}$ e $y_{i}$ representam códigos ortogonais de espalhamento espectral distintos e $L$ é o comprimento total da matriz de códigos Hadamard Walsh.

\subsection{Lógica de Transmissão dos Dados}

As ondas emitidas pelos transponders e recebidas no leitor sofrem dispersões que atenuam a energia do sinal. A fase e amplitude destas ondas são afetadas por questões como a estrutura da antenna, dispersão do sinal e multi-percurso. Poratanto a influência destas características do sistema na fase e amplitude do sinal também descrevem efeitos na transmissão e recepção dos dados, que por sua vez dependem diretamente das impedâncias utilizadas nas emissões do sistema. Como, por exemplo, a impedância da antena $Z_{\text {ant }}$ e a impedância de carga $Z_{\text {load }}$ inerentes aos respectivos transponders.

Assumindo que $Z_{\text {load }}$ pode adotar dois valores $\left(Z_{1}\right.$ e $\left.Z_{2}\right)$, então a dispersão pode ser alterada modificando a impedância de carga do transponder de acordo com os bits a serem 
transmitidos. Portanto podemos manipular os bits enviados a partir das impedâncias de carga $Z_{1}$ e $Z_{2}$. A Figura 8 ilustra a comunicação entre o leitor RFID CDMA e os transponders dispostos no raio de propagação do emissor.

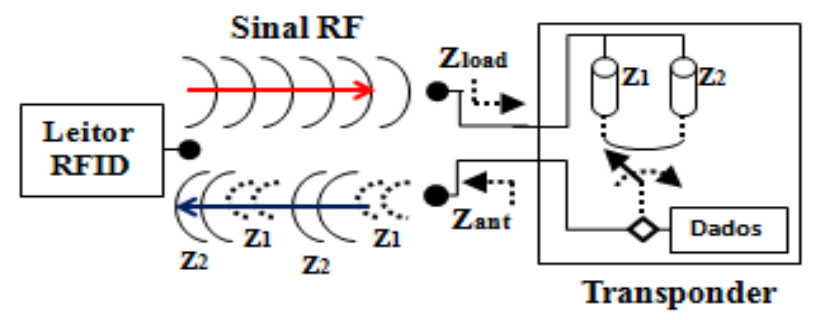

Figura 8: Funcionamento Básico de um Transponder RFID com CDMA.

Os dados são encaminhados para uma de controle que aciona o modulador do transponder com diferentes impedâncias de carga e consequentemente diferentes coeficientes de reflexão. Consideremos $Z_{2}=1$ e a impedância de carga $Z_{1}=0$. A alternância entre $Z_{1}$ e $Z_{2}$ muda o coeficiente de reflexão que por sua vez leva a alterações na fase e/ou amplitude da onda emitida.

Com a inserção da multilinearidade no processo de recepção dos dados, será agregado ao leitor RFID características de unicidade que possibilitarão a distinção fidedigna, para um intervalo limitado de tags. Esta característica será garantida desde que os princípios da decomposição por fatores paralelos, descritos neste trabalho, sejam respeitados e implementados ao sistema.

\section{RESULTADOS DE SIMULAÇÃO}

Apresentaremos a seguir algumas simulações em software para ilustrar o desempenho da arquitetura RFID CDMA PARAFAC proposta neste trabalho. É sabido que a partir do conhecimento prévio de uma das matrizes que compõem a estrutura tensorial podemos estimar as demais dimensões do modelo tensorial, portanto geramos estocasticamente a matriz de símbolos que compõe o modelo e simulamos resultados a partir do $\mathrm{MMC}$ (Método de Monte Carlo) utilizando parâmetros reais para sistemas RFID normatizados.

Os dados de cada transponder são concatenados as respectivas sequências de espalhamento de espectro, Sn, e enviados ao leitor RFID, que por sua vez aplica os métodos de estimação e algoritmo PARAFAC para obter as informações estimadas de cada transponder. 0 produto da informação estimada por $S_{n}^{-1}$ resulta na informação original estimada sem espalhamento de espectro. A Figura 9 representa a transmissão simultânea de 4 (quatro) transponders ativos e o uso do tratamento tensorial no receptor (Portal Leitor RFID) através da decomposição por fatores paralelos.

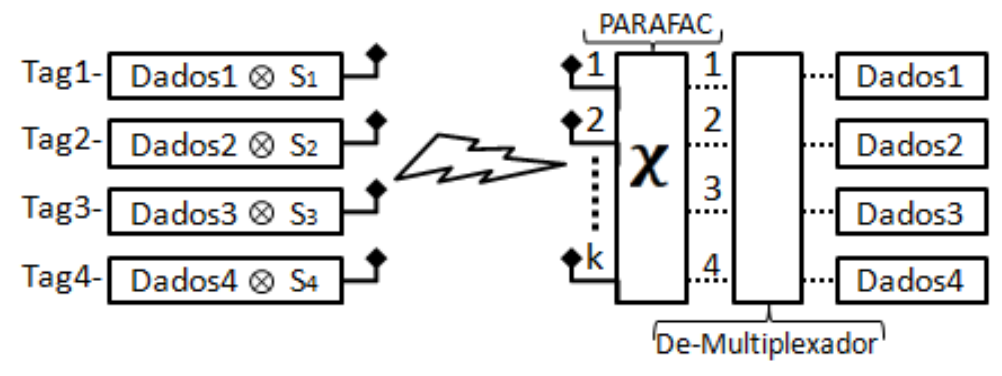

Figura 9: Transmissão RFID CDMA PARAFAC. 
onde $S_{1}, S_{2}, S_{3}$ e $S_{4}$ são respectivamente as sequências da matriz de Hadamard Walsh $\left(H_{4}\right)$ para o espalhamento do espectro em banda larga.

Consideremos possuir o conhecimento da Matriz de Assinaturas Espaciais ( $A$ ) conforme a disposição estática dos transponders em relação ao portal RFID. Portanto assumiremos que esta matriz é conhecida no receptor e desta forma utilizaremos o algoritmo ALS-PARAFAC para realizar a estimação das matrizes de códigos CDMA Hadamard-Wash (C) e Sinais dos Transponders (S) a partir dos seguintes passos descritos no Quadro 2.

\section{Quadro 2. Algoritmo ALS-PARAFAC $\rightarrow$ RFID CDMA}

1. Inicialize $S$ aleatoriamente;

2. Atualizando A:

$$
A^{\top}=(C \diamond S)+X_{(3)} ;
$$

3. Atualizando S:

$$
S^{\top}=(A \circ C)+X_{(2)}
$$

4. Repetir os Passos 2-3 até a convergencia.

\subsection{Método de Monte Carlo Aplicado ao Modelo RFID CDMA PARAFAC.}

Os dados de entrada foram gerados estocasticamente a partir da criação aleatória das matrizes de símbolos transmitidos por cada transponder ativo. Então essas matrizes são usadas para alimentar o modelo ALS-PARAFAC e por sequência o NMSE para geração das respostas estimação dos dados. As matrizes geradas são analisadas estatisticamente para quantificar o quão ótima é a estimação do modelo. O método de Monte Carlo é aplicado ao nosso modelo seguindo os passos descritos no Quadro 3.

Quadro 3. Método de Monte Carlo $\rightarrow$ Matriz de Símbolos

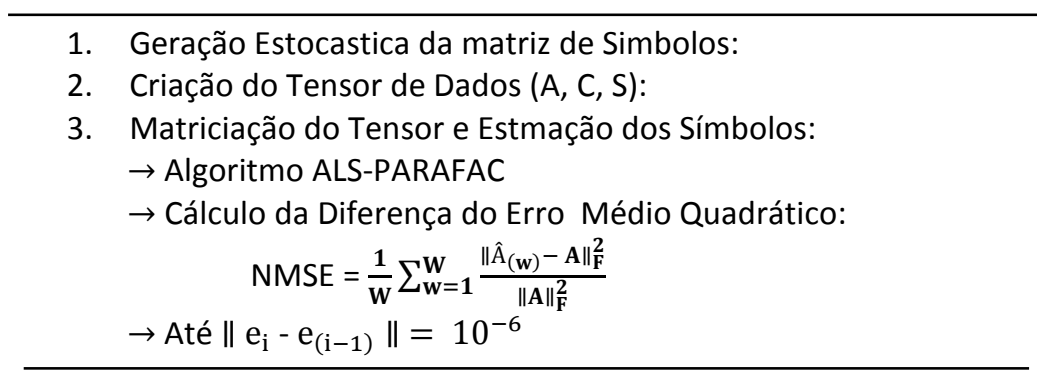

\subsection{Estimação das Matrizes de Símbolos.}

A Figura 10 ilustra o desempenho da estimação da matriz S (Sinais dos Transponders) através da análise da Taxa de Erro de Bit (BER) para diferentes valores do número de antenas receptoras (AntRx), considerando fixo e igual a 4 o número de transponders ativos, para diversos valores de Relação Sinal Ruído (SNR). Os resultados mostram claramente que a BER é menor quando utilizamos um número maior de antenas receptoras no portal RFID CDMA PARAFAC em relação ao diferentes valores de SNR.

A Figura 11 ilustra o desempenho da estimação da matriz " $\mathrm{S}$ " através da análise do NMSE (Normalized Mean Square Error) para valores de SNR de $-5 \mathrm{~dB}$ até $25 \mathrm{~dB}$. Nesta simulação matemos fixo e igual a 4 o número de transponders ativos e variamos o número de antenas receptoras no leitor RFID CDMA PARAFC em cada simulação, assumindo AntRx $=4,8$ e 15 . Os resultados obtidos para a estimação da diferença do erro quadrático entre a matriz de símbolos 
real e a estimada demonstram, conforme esperado, que as curvas sofreram variações sensíveis à medida que houve o aumento do número de AntRx e a melhora da SNR. Concluímos dessa forma que o desempenho da estimação da matriz de Sinais dos Transponders depende diretamente do número de antenas utilizadas no receptor RFID CDMA PARAFAC.

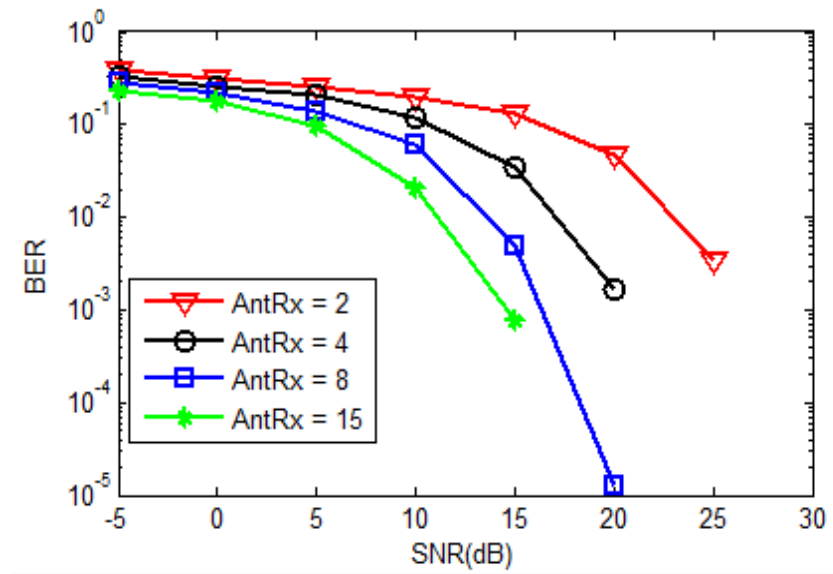

Figura 10: BER por SNR para Tags $=4$ e AntRx $=2,4,8 \mathrm{e}$ 15.

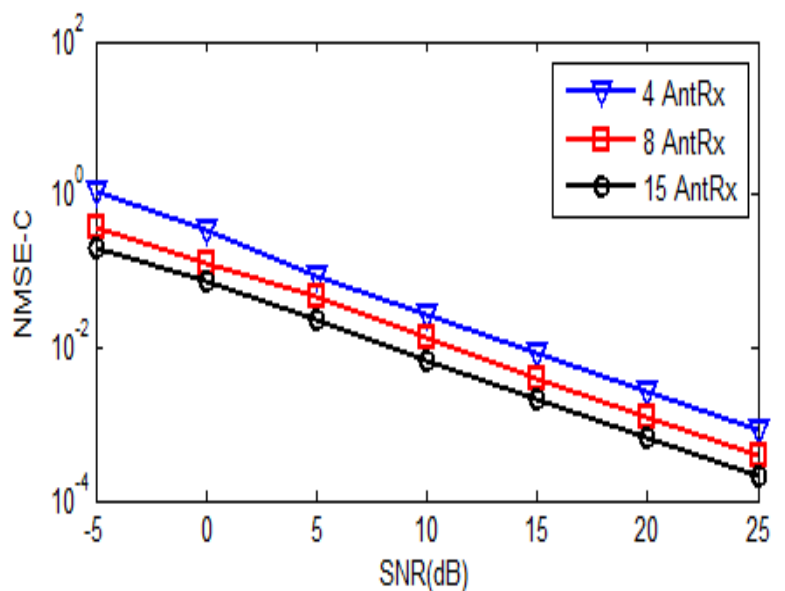

Figura 11: NMSE-C por SNR para Tags $=4$ e AntRx= 4, 8 e 15.

A Figura 12 ilustra o desempenho da estimação da matriz de sinais dos transponders através do NMSE. Nesta simulação foi assumido que o número de antenas receptoras no portal e o número de transponders são constantes e iguais a 4 . Nesta simulação foi analisado o desempenho da estimação da matriz variando o número de Snapshots para valores iguais a $\mathrm{N}=25$, 50 e 100 e valores de SNR variando de $-5 \mathrm{db}$ até $25 \mathrm{db}$.

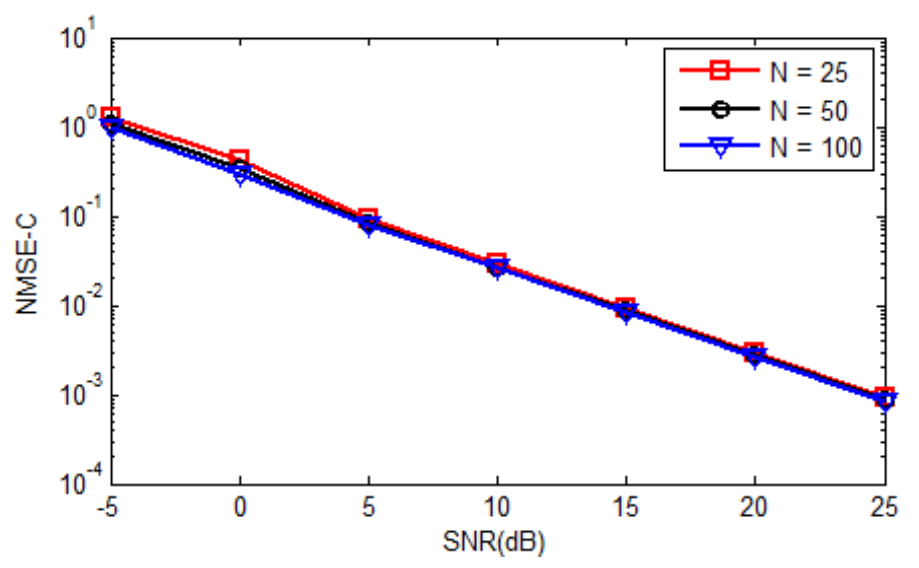

Figura 12: NMSE-S vs. SNR (dB) para $K=4, M=4$ e $N=25 ; 50$ e 100.

Os resultados obtidos demonstram que as curvas ficam praticamente sobrepostas, evidenciando que não haverá uma diferença significativa na estimação dos símbolos para variação do número de Snapshots para o cenário descrito neste trabalho. Por definição Snapshot é o estado de um sistema em um determinado ponto no tempo, este parâmetro para nossa aplicação representa o número de símbolos que é transmitido por pacote por cada tag. Entretanto sabemos que o custo computacional de operação do sistema é proporcional à quantidade de símbolos transmitidos, embora não haja sensíveis varações quanto à estimação de erros. 
A Figura 13a mostra uma comparação entre o número de iterações necessárias para obtermos o nível aceitável de estimação considerando fixos e iguais a 4 (quatro) os valores de AntRx e Tags e variando os valores de Snapshots. Há variações consideráveis do número de iterações até SNR igual a $5 \mathrm{~dB}$, entretanto o aumento da Relação Sinal Ruído a partir de $5 \mathrm{~dB}$ faz com que haja a convergência ótima para os diferentes valores de snapshots.

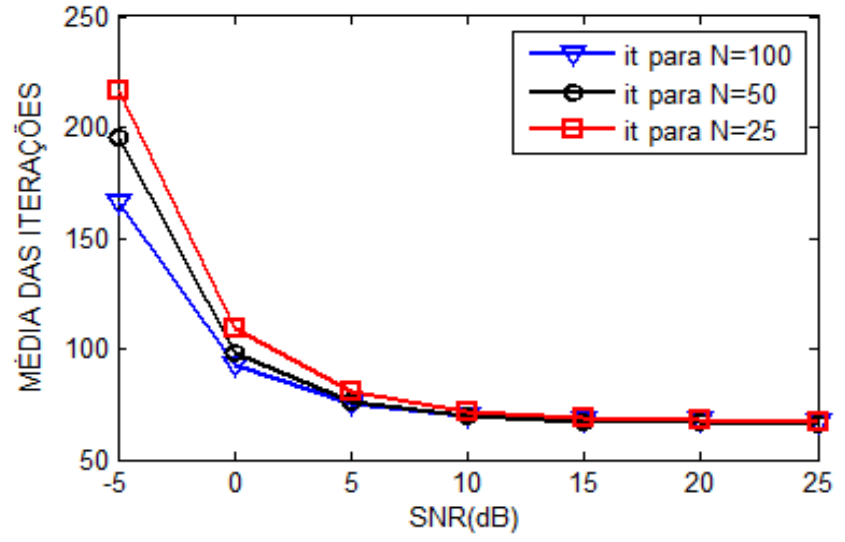

Figura 13.a. Média das Iterações vs. SNR (dB) para AntRx = 4 e $\mathbf{N}=25 ; 50$ e 100.

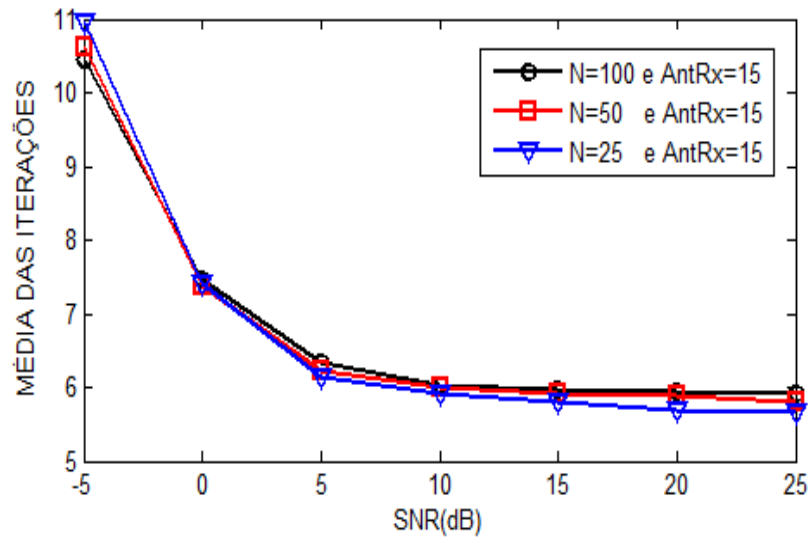

Figura 13.b. Média das Iterações vs. SNR (dB) para AntRx = 15 e $\mathbf{N}=25 ; 50$ e 100.

Uma solução satisfatória para a redução do número de iterações necessárias para a convergência do modelo é o aumento número de AntRx do portal receptor. Esta ação diminui exponencialmente o número de iterações necessárias para o algoritmo chegar ao nível aceitável de estimação, erro de $10^{-3}$. Percebe-se que mesmo com uma SNR bem baixa, $-5 \mathrm{~dB}$, o número de iterações foi reduzido a aproximadamente 0,05 do número de iterações para 4 AntRx. A Figura 13b mostra o desempenho do sistema para a mesma variação de Snapshots da Figura 13a, mas para um cenário com 15 AntRx.

A Figura 14a ilustra o desempenho da estimação da matriz de Símbolos dos Transponders em termos de Taxa de Erro de Bit, BER, considerando fixos e iguais a 4 os valores de AntRx e Tags. Nesta simulação o desempenho da estimação é analisado para diversos valores de SNR, utilizando as modulações M-QAM e M-PSK para diversos índices.

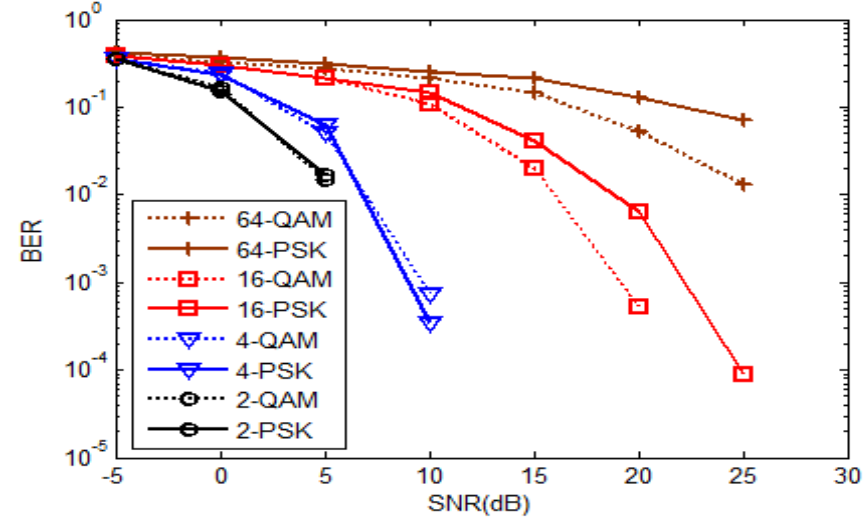

Figura 14.a. BER vs. SNR (dB): Comparação N-QAM vs. N-PSK.

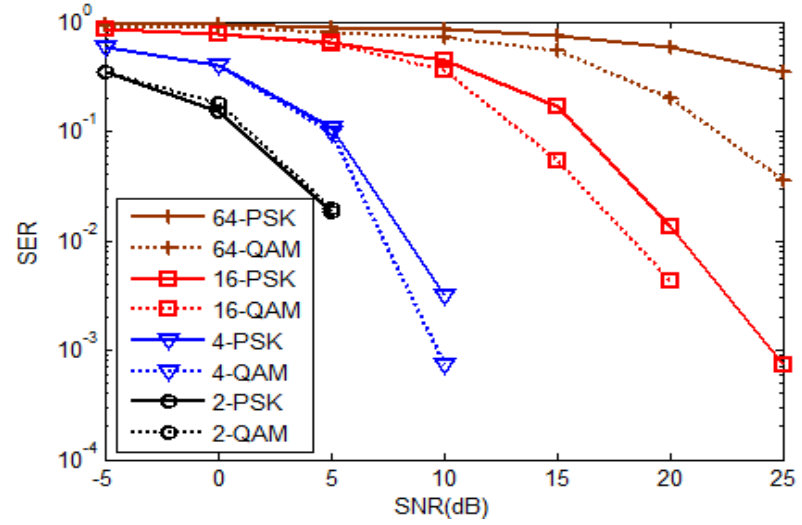

Figura 14.b. SER vs. SNR (dB): Comparação N-QAM vs. N-PSK.

Observa-se que os resultados obtidos apresentam um aumento na BER à medida que o índice de modulação aumenta, além disso, observa-se que para os casos 2-QAM e BPSK e 4-QAM 
e 4-PSK a BER não varia quando a modulação é modificada para o mesmo índice de modulação, este comportamento é esperado em virtude da distância entre os símbolos ser praticamente a mesma. Porém para altos índices de modulação esse aumento da BER é mais perceptível, ou seja, a modulação PSK apresenta uma taxa de erro maior, pois a distância entre os símbolos é menor em comparação com a modulação QAM.

Na Figura 14b temos a SER para o mesmo cenário citado anteriormente. Os resultados para este parâmetro são semelhantes aos resultados obtidos para a BER vs. SNR (dB), entretanto apresenta-se um aumento na SER à medida que o índice de modulação aumenta, sendo este fato oriundo praticamente da distância entre os símbolos em cada caso. É importante salientar que para o caso BPSK a BER é igual a SER, pois cada símbolo é representado por apenas um bit. Para altos índices de modulação a modulação PSK apresenta uma maior SER em comparação com a modulação QAM, pois à distância entre os símbolos nessa modulação diminui, apresentando com isso uma maior sensibilidade ao ruído.

\section{CONCLUSÃO}

Realizamos as análises de desempenho da estimação das matrizes de Símbolos para diversos canários, usufruindo dos benefícios da álgebra multilinear no que se refere ao tratamento tensorial de dados. O modelo é baseado num arranjo de antenas receptoras com processamento inteligente e com modelagem tensorial. Para a realização da estimação das matrizes de Símbolos foi utilizado o algoritmo ALS-PARAFAC em múltiplos cenários distintos e através dos resultados obtidos percebe-se que este algoritmo pode ser utilizado na realização das estimações de uma arquitetura RFID com CDMA com uso simultâneo e limitado de múltiplas tags. Através dos resultados obtidos foi possível realizar uma análise do desempenho da estimação para diversos parâmetros diferentes, viabilizando um estudo do comportamento do modelo RFID CDMA PARAFAC proposto nesta pesquisa. Além disso, percebe-se que os resultados estão de acordo com a literatura, o que torna a utilização de modelos tensoriais associados a métodos probabilísticos de cunho estocásticos perfeitamente aceitáveis em problemas que envolvem estimação de parâmetros e que necessitam que a propriedade de unicidade seja garantida.

\section{REFERÊNCIAS BIBLIOGRÁFICAS}

1. Alamouti, S. A. Simple transmit diversity technique for wireless communications. (1998).

2. Almeida, A. L. F. Tensor Modeling and Signal Processing for Wireless Communication Systems. (2007).

3. Bro R., Sidiropoulos N. D. \& Giannakis, G. B. Optimal joint azimuth-elevation and signal-array response estimation using parallel factor analysis. (1998).

4. Carroll, J. D. \& Chang, J. Analysis of individual differences in multidimensional scaling via as nway generalization of young decomposition. (1970).

5. Cichocki A. \& Zdunek R., Nonnegative Matrix and Tensor Factorizations - Applications to Exploratory Multi-way Data Analysis and Blind Souce Separation. (2009).

6. Finkenzeller K., RFID Handbook: Fundamentals and Applications in Contactless Smart Cards 
and Identification. (2003).

7. Foschini, G. J. \& Gans M. J. On Limits of Wireless Communications in a Fading EnvironmentWhen Using Multiple Antennas .(1998)

8. Fusco, V. F. Teoria e Técnicas de Antenas: Princípios e Prática. (2006).

9. Hansen, R. Relationships between antennas as scatterers and as radiators. (1989).

10. Harshman, R. A. Foundations of the parafac procedure: Model and conditions for na explanatory multi-mode factor analysis. UCLA Working Papers in Phonetics. (1970)

11. Haykin S. \& Moher M., Sistemas modernos de comunicações wireless. (2008).

12. Kolda, G. T. \& Bader, B. W. Tensor decompositions and applications. Society for Industrial and Applied Mathematics. (2009).

13. Kroonenberg, P. M. Applied Multiway Data Analysis. (2008).

14. Kruskal, J. B. Three-way arrays: rank and uniqueness of trilinear decomposition, with applications to characterization of probabil- ity distributions. Linear Algebra Application. (1977).

15. Lacerda Neto, R. L. Receptores MIMO Baseados em Algoritmo de Decomposição PARAFAC. (2005).

16. Loeffler A. \& Friedrich A. Using CDMA as Anti-Collision Method for RFID - Research \& Applications. (2003).

17. Marques, C. A. A tecnologia RFID na logística interna industrial: pesquisa exploratória numa empresa de usinados para o setor aeroespacial. (2008).

18. Mota, R. P. Extensões ao protocolo de comunicação EPC Global para tags Classe 1 utilizando autenticação com criptografia de baixo custo para segurança em identificação por radiofrequência. (2006).

19. Mutti. C \& Floerkemeier C. CDMA-based RFID Systems in Dense Scenarios: Concepts and Challenges. (2008).

20. Nikitin, P. \& Rao, K. Antennas and propagation in uhf rfid systems, RFID. (2008).

21. Rong, Y. Blind signal spatial siagnature estimation using parafac model. Master's thesis, Gerhard Mercator University (2002).

22. Sidiropoulos, N. D. \& Dimic, G. Z. Blind multiuser detection in WCDMA systems with large delay spread. (2004).

23. Sidiropoulos, N. D. \& Liu, X. Parafac techniques for highresolution array processing. (2000).

24. Smilde, A., Bro R. \& Geladi, P. Multi-way Analysis - Applications in the Chemical Sciences. (2004). 